\title{
Kinetics of Phenylalanine Disappearance after Intravenous Load in Phenylketonuria and Its Genetic Variants
}

\author{
FRANÇOISE REY, ${ }^{(33)}$ FÉLICIENNE BLANDIN-SAVOJA, AND JEAN REY \\ Unité de Recherches de Génétique Médicale (INSERM), Hôpital des Enfants-Malades, Paris, France
}

\section{Summary}

Intravenous phenylalanine loading tests were performed in 22 children affected by different types of hyperphenylalaninemia: six cases of classical phenylketonuria (PKU), six cases of atypical PKU, five cases of mild permanent hyperphenylalaninemia, three cases of "transient" PKU, and two cases of the "new variant with progressive neurological illness resistant to dietary treatment," one of them suffering from DHP reductase deficiency and the other being affected by a defect in biopterin synthesis. A $0.06 \mathrm{M}$ L-phenylalanine $\left(500 \mathrm{ml} / \mathrm{m}^{2}\right)$ solution in $\mathrm{NaCl}$ was perfused during $3 \mathrm{hr}$ so that a plasma level of about $2-3 \mu \mathrm{mol} \cdot \mathrm{ml}^{-1}$ was obtained. Results were compared to those obtained in five controls.

In classical, atypical, and mild types, a first order kinetics was observed; the constant of the disappearance rate $(k)$ was, respectively, $2.5,4.6$, and $8.5 \times 10^{-4} \cdot \mathrm{min}^{-1}$, the differences being highly significant $(P<0.001)$. Expressed as a function of the value of $k$ found in controls $\left(1.37 \times 10^{-2} \cdot \mathrm{min}^{-1}\right)$, these figures represent, respectively, $1.8,2.4$, and $6.2 \%$ of the normal. A first order kinetics was observed also in the new variant, the values of $k$ (3.5 and 4.4 $\times 10^{-4} \cdot \mathrm{min}^{-1}$ ) being similar to those found in atypical PKU. On the other hand, in the so-called transient PKU, the kinetics of disappearance is a zero-order one. Moreover, the time course of fall is, on an average, $48 \mathrm{hr}$, thus demonstrating the persistance of a metabolic disorder in spite of the subnormal plasma levels $\left(0.2-0.3 \mu \mathrm{mol} \cdot \mathrm{ml}^{-1}\right)$ on a regular diet.

The results demonstrate that in the common variants (classical PKU, atypical PKU, and mild forms) the disappearance rate constant of phenylalanine after an intravenous perfusion is proportional to the residual hydroxylase activity as assayed in liver biopsies. They suggest that transient PKU, which is, in fact, a permanent condition, might result from the accumulation of an intermediate, the concentration of which remains constant during the hydroxylation process. Finally, in spite of a normal hydroxylase activity in the in vitro system, an in vivo hydroxylation rate of 3 and $4 \%$ of the normal was found in the two children affected by the new variant, which is in good agreement with their daily tolerance and their fasting phenylalanine plasma level on a regular diet.

\section{Speculation}

The demonstration of a zero-order kinetics of phenylalanine disappearance in transient PKU suggests the accumulation of an intermediate released during the hydroxylation process, which might occur from a phenylalanine hydroxylase-stimulating protein defect.

Phenylalanine, administered to normal individuals as an intravenous load, is metabolized by a first order reaction, the semilog plot of the phenylalanine concentration against time giving a straight line within the appropriate limits (28). The kinetics of disappearance of phenylalanine obeys also to first order kinetics in heterozygotes for classical PKU. Its rate of fall, expressed as a first order rate constant $(\mathrm{k})$, is, in that case, exactly half of that of homozygotes for the wild-type gene, suggesting thus that $\mathrm{k}$ is related to the amount of enzyme in liver $(5,28)$. On the other hand, a faster return of plasma phenylalanine concentration to normal values with a phenylalanine-restricted diet $(25)$ or to the fasting level after an oral load $(4,12)$ in hyperphenylalaninemia than in PKU has been proved and an atypical curve of disappearance, linear with time, that is independent of the blood phenylalanine concentration (zero-order kinetics), has been obtained in the mothers of two unrelated children affected by a variant form of hyperphenylalaninemia. These subjects have been considered to possess a "third allele" at the phenylalanine hydroxylase locus $(28,29)$. However, up to now, owing to the moderate rise in phenylalanine plasma level and/or the too short duration of follow-up, the kinetics of injected phenylalanine has never been correctly analyzed in PKU and no difference in the rate of phenylalanine disposal between PKU and PKU variants has been demonstrated by this technique $(5,14)$.

Therefore, by using a prolonged perfusion method which allows, with the same amount of phenylalanine, reaching higher levels (up to $2-3 \mu \mathrm{mol} \cdot \mathrm{ml}^{-1}$ ) than those obtained by oral loading tests, and by following phenylalanine disappearance until equilibrium (or at least during 3 days in classical PKU), we have attempted to find some difference in the type of kinetics among the PKU variants, and to verify whether the in vivo phenylalanine disappearance rate actually reflects the residual activity of the hydroxylase system. Moreover, this technique was used to study the effect of raised plasma phenylalanine levels on biopterin metabolism in "hyperphenylalaninemia with normal hydroxylase activity" (23).

\section{MATERIALS AND METHODS}

\section{SUBJECTS}

Twenty-two patients ( 7 females and 15 males), ranging in age from 3 months to 12 years, were studied; hyperphenylalaninemia was detected in 19 at birth and, in the remaining 3, it was recognized at older ages in the course of familial investigations. They were classified in function of their phenylalanine plasma level with a diet containing $3.5 \mathrm{~g}$ protein $/ \mathrm{kg}$ body wt at the moment of the initial diagnosis: nine, in whom phenylalanineblood concentration exceeded $1.5 \mu \mathrm{mol} \cdot \mathrm{ml}^{-1}$ in these conditions, were considered as affected by classical PKU; eight in whom it ranged between 0.9 and $1.5 \mu \mathrm{mol} \cdot \mathrm{ml}^{-1}$ by atypical PKU, and five in whom it was below $0.9 \mu \mathrm{mol} \cdot \mathrm{ml}^{-1}$ by mild permanent hyperphenylalaninemia according to the terminology of Auerbach et al. (1). In 14 cases the diagnosis was confirmed by phenylalanine load at 6 months or 1 year of age, thus providing, with the three not detected at birth, six patients suffering from classical PKU, six from atypical PKU, and five from mild hyperphenylalaninemia. In three cases which were initially classified as classical (A. L.) or 
atypical PKU (F. T. and Y. L.) and treated for a few months with Lofenalac, phenylalanine plasma level did not increase above $0.2-0.3 \mu \mathrm{mol} \cdot \mathrm{ml}^{-1}$ after reintroduction of a normal diet between 4 and 6 months of age and stayed thereafter within this range; they are called transient PKU. It is worthwhile noting that, in contrast to common variants, neither elevated fasting phenylalanine plasma level nor $\mathrm{P} / \mathrm{T}$ molar ratio increase was found in both parents of these children. In the three cases, intravenous load was performed 6 months after apparent recovery. The last two children, classified first as classical PKU, are affected by the "new variant of PKU with progressive neurological illness unresponsive to phenylalanine restriction" (26). In both children, phenylalanine hydroxylase activity (EC. 1.14.16.1) assayed on needle liver biopsies was found in the normal range, dihydropteridine reductase (EC. 1.6.99.7) being absent in one case (D. P.) (23) and normal in the other one affected by a defect in biopterin metabolism (C. A.) $(20,22)$. In addition, five normal children ranging in age from 1 to 4 years, hospitalized in the metabolic ward for another reason, were tested as controls. In all cases informed consent was obtained from the parents (Table 1).

\section{PHENYLALANINE LOADING TEST}

Intravenous perfusion was performed after an overnight fast with a $0.06 \mathrm{M}$ solution of pure L-phenylalanine (Sigma) dissolved in saline $0.15 \mathrm{M}$ and sterilized by passage through a $0.22 \mu$ Millipore filter. Assuming a rapid equilibrium between cellular and extracellular fluids and a liquid compartment of $65 \%, 500$ $\mathrm{ml} / \mathrm{m}^{2}$ were infused at constant rate flow during $3 \mathrm{hr}$; only onehalf of a dose was perfused in $\mathrm{l} \mathrm{hr}$ in the two patients (M. H. and V. O.) who were, respectively, $12 \%$ and 9 years old. The diet was unchanged during the weeks before the intravenous load and the following days, the phenylalanine daily intake being restricted, if necessary, in order to keep fasting blood-phenylalanine concentrations within 0.2 and $0.5 \mu \mathrm{mol} \cdot \mathrm{ml}^{-1}$.
In classical and atypical PKU, blood samples were collected at the end of the infusion, $6 \mathrm{hr}$ later, and afterwards twice a day at $6 \mathrm{AM}$ and 6 PM during 3 or 4 days. In mild type, blood collections were discontinued $48 \mathrm{hr}$ later; the same protocol was used for transient PKU in whom analyzes were done every 4 or $6 \mathrm{hr}$ during the first day. In the controls, except the first case for whom blood collection took place unfortunately only $6 \mathrm{hr}$ afterwards, phenylalanine plasma levels were measured every hour for $4 \mathrm{hr}$ or at 2 , 4 , and $6 \mathrm{hr}$. Plasma phenylalanine was measured on microsamples $(10 \mu \mathrm{l})$, in triplicate, by automatic fluorimetry (Technicon AutoAnalyzer) according to the technique of Hill et al. (13).

The apparent rate constant of the first order kinetics (k) was calculated from the general equation $\mathrm{d} \phi / \mathrm{dt}=-\mathrm{k}(\phi-\phi 0)$ where $\phi$ is the concentration of phenylalanine at time $(\mathrm{t})$ and $\phi 0$ is its postinjection concentration (zero time). Owing to the inaccuracy of the fluorimetric method below $0.2 \mu \mathrm{mol} \cdot \mathrm{ml}^{-1}$, the fasting phenylalanine concentration $(\phi 0)$ was not deduced from $\phi$ for setting out the figures; lines were drawn by inspection. The linearity of the relation of $(\phi-\phi 0)$ to $t$ in transient PKU was established from the sums of square deviations (28).

\section{RESULTS}

\section{CLASSICAL AND ATYPICAL PKU}

In the six cases of classical PKU, phenylalanine plasma level increased in $3 \mathrm{hr}$ from an initial value of $0.37 \pm 0.02$ to $3.24 \pm$ $0.70 \mu \mathrm{mol} \cdot \mathrm{ml}^{-1}$. In the same time it increased, in the six cases of atypical PKU, from $0.61 \pm 0.31$ (two of them were untreated cases) to $2.49 \pm 0.66 \mu \mathrm{mol} \cdot \mathrm{ml}^{-1}$. This lower last value can be explained by the administration of only one-half of a dose to the two oldest children. The phenylalanine plasma level reached 2.77 $\pm 0.67 \mu \mathrm{mol} \cdot \mathrm{ml}^{-1}$ in the four other atypical cases, a value which is not significantly different from that observed in classical PKU.

Table 1. Clinical data and diet of hyperphenylalaninemic patients at the time of the intravenous phenylalanine loading test

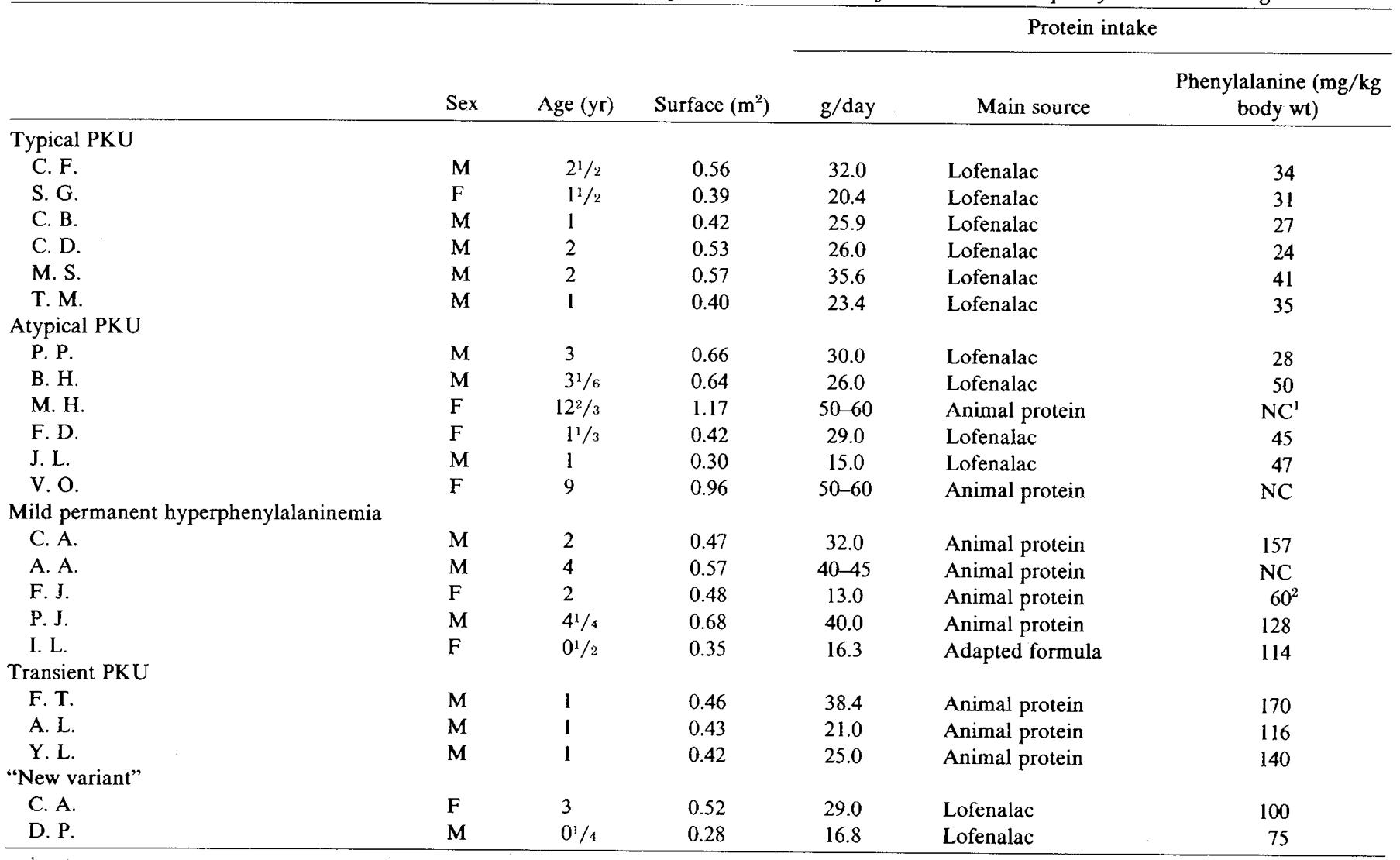

${ }^{1} \mathrm{NC}$, non strictly controlled.

${ }^{2}$ Reduced intake due to anorexia. 
The phenylalanine disappearance rate was, in all cases, proportional to the substrate concentration, following an exponential kinetics (first order). Values of $\mathbf{k}$, calculated from the slope on the straight line obtained by plotting logarithmic concentration of plasma phenylalanine versus time, were $2.5 \pm 0.7 \times 10^{-4} \cdot \mathrm{min}^{-1}$ for classical PKU (Fig. 1) and $4.6 \pm 1.9 \times 10^{-4} \cdot \mathrm{min}^{-1}$ for atypical cases, including 1 patient (F. D.) who vomited after the phenylalanine load and had a slightly faster disappearance rate $(\mathrm{k}=8.1$ $\times 10^{-4} \cdot \mathrm{min}^{-1}$ ) (Fig. 2 ). These values corresponded, respectively, to 1.8 and $3.4 \%$ of the normal activity $\left(1.37 \pm 0.45 \times 10^{-2} \cdot \mathrm{min}^{-1}\right)$. The difference between classical and atypical slopes of disappearance is highly significant $(P<0.001)$.

\section{MILD HYPERPHENYLALANINEMIA}

In the five children affected by mild hyperphenylalaninemia, phenylalanine serum level increased from $0.29 \pm 0.09$ to $3.05 \pm$ $0.28 \mu \mathrm{mol} \cdot \mathrm{ml}^{-1}$. As in classical and atypical PKU, phenylalanine concentrations, when plotted against time, exhibit a first order kinetics. Value of $\mathrm{k}$, calculated for all children but one (F. J.)

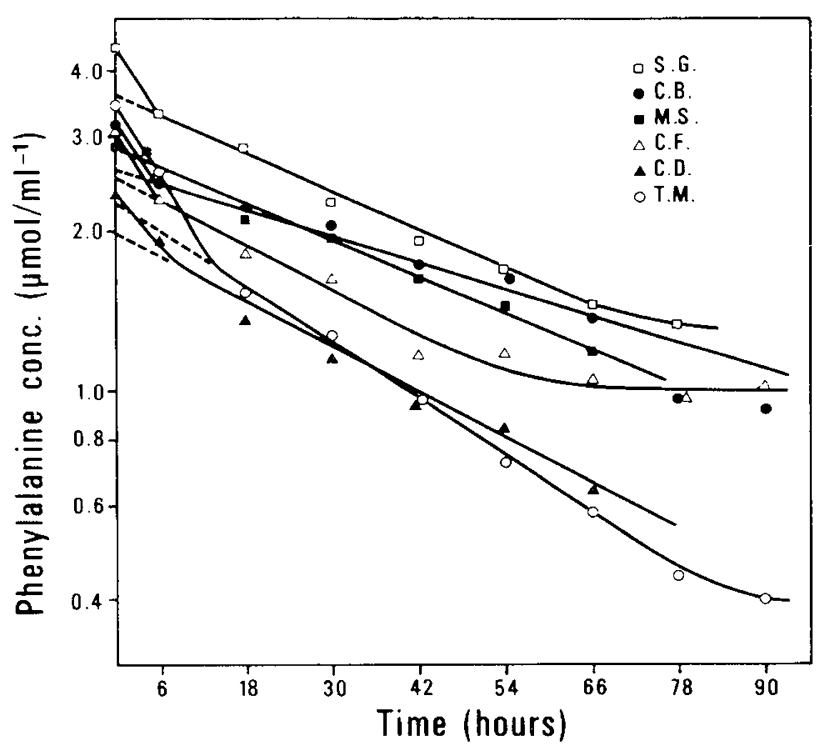

Fig. 1. Kinetics of phenylalanine disappearance in classical PKU (semilog plot of phenylalanine concentration as a function of time). Initials refer to Table 1 .

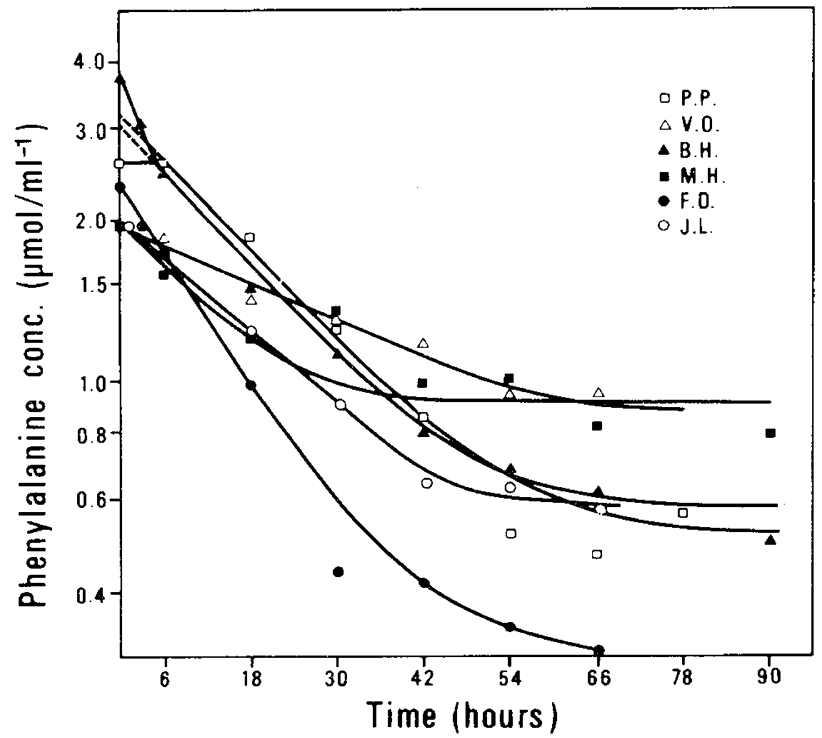

Fig. 2. Kinetics of phenylalanine disappearance in atypical PKU (same symbols as in Fig. 1).

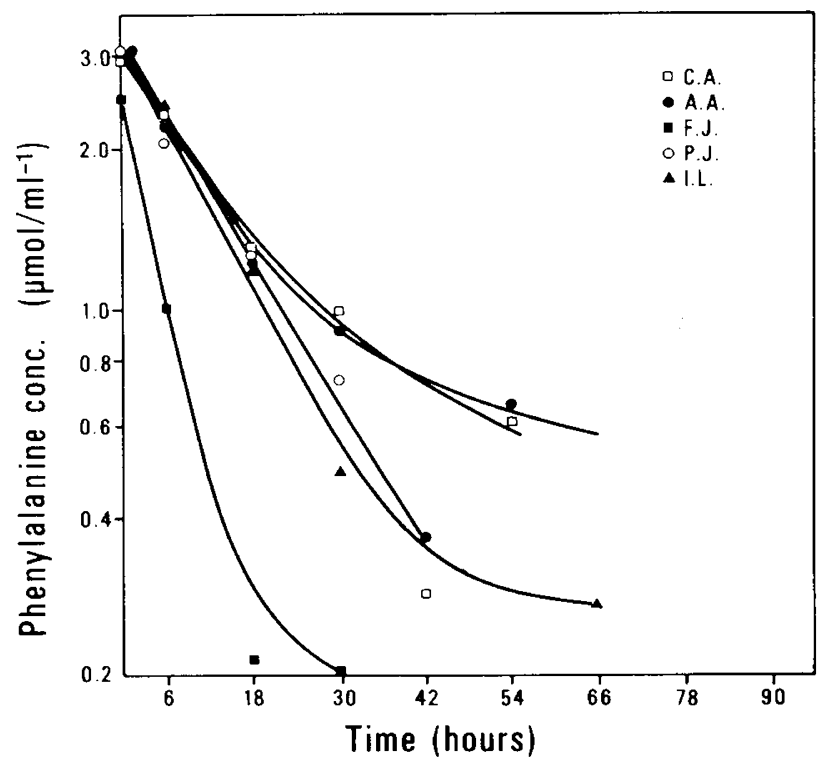

Fig. 3. Kinetics of phenylalanine disappearance in mild permanent hyperphenylalaninemia (same symbols as in Fig. 1).

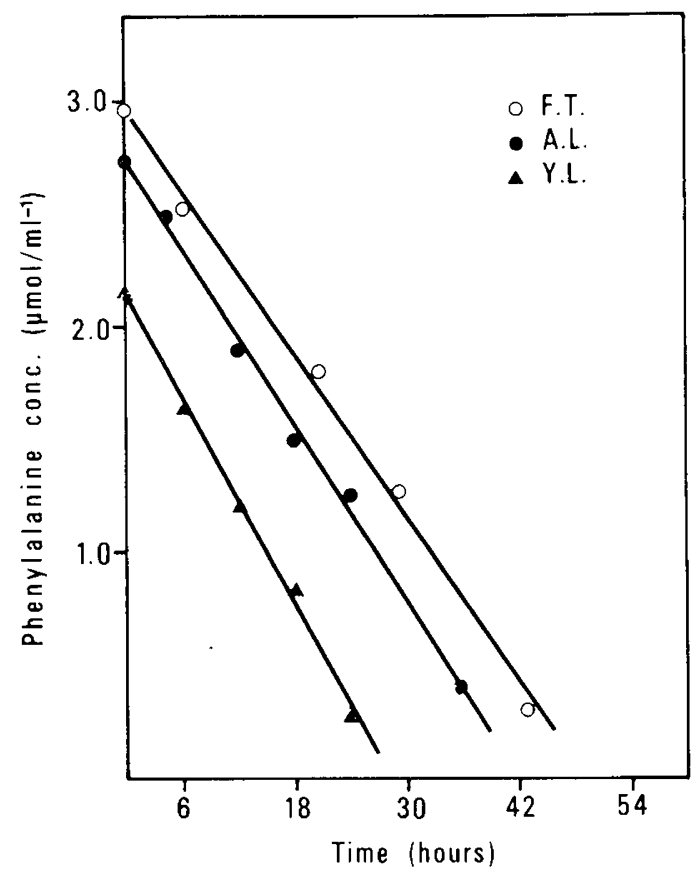

Fig. 4. Kinetics of phenylalanine disappearance in "transient" PKU (phenylalanine concentration vs. time). Initials refer to Table 1.

having a higher value of $\mathbf{k}$ due to anorexia and thus inadequate protein intake during the test, was $8.5 \pm 1.5 \times 10^{-4} \cdot \mathrm{min}^{-1}$ (i.e., $6.2 \%$ of control subjects). This value is significantly different $(P$ $<0.001$ ) from that of atypical cases (Fig. 3).

\section{TRANSIENT PKU}

The three children suffering from transient PKU all showed a similar atypical pattern when discharging phenylalanine load. Indeed, their plasma concentrations came back from $2.60 \pm 0.41$ $\mu \mathrm{mol} \cdot \mathrm{ml}^{-1}$ to fasting levels within 2 days as in a mild hyperphenylalaninemia, but the rate of disappearance was constant for any concentration of phenylalanine, carrying out a zero-order kinetics (Fig. 4). The mean square deviation from linear regression of the phenylalanine concentration against time compared with the variance due to random error determination gave a nonsignificant deviation from linearity in every case. 
HYPERPHENYLALANINEMIA RESISTANT TO DIETARY TREATMENT

A first order kinetics of disappearance was found in two patients affected by the new variant, the value of $\mathrm{k}$ being $3.5 \times 10^{-4} \cdot \mathrm{min}^{-1}$ in the case where both hydroxylase and reductase activities are normal (C. A.) and $4.4 \times 10^{-4} \cdot \mathrm{min}^{-1}$ for the child affected by a dihydropteridine reductase deficiency (D. P.). Biopterin values in plasma and urines in response to phenylalanine challenge were reported elsewhere (23).

\section{DISCUSSION}

Intravenous phenylalanine load has been used by many investigators as a test of differentiation between normals (homozygotes for the wild-type gene) and heterozygotes for the wild-type gene and the gene for the classical type of phenylketonuria ("classic" heterozygotes), but it has failed, so far, to improve the discriminatory power of oral loads or that of the fasting phenylalanine to tyrosine $(\mathrm{P} / \mathrm{T})$ molar ratio. It clearly demonstrated, however, that the rate of disappearance of administered phenylalanine was, on an average, exactly twice as fast in normals than in classic heterozygotes, thus suggesting that the latter has only half of the amount of the normal enzyme. Therefore, this technique makes possible an in vivo estimation of the residual hydroxylating system activity in the different PKU variants, insofar as 1) protein intake and aminoacid incorporation into the proteins remain constant along the test, and 2) there is no difference between variants neither with regard to transaminase activity (22) nor with phenylalanine urinary excretion (11). Owing to the too short duration of the experiments, such a possibility to differentiate between PKU variants and to characterize their kinetics of disappearance has not yet given conclusive results, if not the zero-order kinetics found by Woolf et al. $(28,29)$ in the mothers of two unrelated children affected by transient PKU and in two other parents of children with PKU, describing thus a third allele at the phenylalanine hydroxylase locus.

The data that we have obtained with the modified technique described above demonstrate that phenylalanine is metabolized by the three common variants (classical PKU, atypical PKU, and mild permanent hyperphenylalaninemia) by a first order kinetics as in normal and classic heterozygotes, their disappearance slopes being significantly different. The increase of phenylalanine plasma level, under which our patients are classified in either group, seems thus a good phenotypic discriminant, the only condition being that protein, and therefore phenylalanine, intake is well defined (e.g., $3 \mathrm{~g} / \mathrm{kg}$ body $\mathrm{wt}$, as in our protocol). Nevertheless, the existence of these three phenotypes does not necessarily mean that they correspond to three distinct genotypes and the probability is very high that a number of these children would not be homozygotes for one mutant gene, but would represent "genetics compounds" (heterozygotes for two mutant alleles). For example, it is impossible to state whether patients affected by atypical PKU are heterozygotes for both "classical" and "mild" genes (classical/ mild) or, on the contrary, whether patients affected by mild permanent hyperphenylalaninemia are heterozygotes for "classical" and "atypical" mutant alleles (classical/atypical) (complementation interallelic), examples of such a mode of inheritance have already been reported $(7,17,21,30)$. Indeed, the three common variants could theoretically result from the cooperation between two mutant alleles; but the fact that, in our series, parents of "atypical patients" are more often originating from Portugal or mediterranean countries than those of children suffering from "mild" or "classical" forms suggests that atypical PKU constitutes a distinct group, as has been pointed out by Efron (8) and provides another possible model involving three alleles.

Even though enzymatic analyzes have not been performed in these patients and, thus, intraindividual correlation for the phenylalanine hydroxylase activities and the phenylalanine disappearance rate is not possible, the relative values of $k$ (patients/controls ratio) in each type are in agreement with the values of hydroxylase activities assayed in liver biopsies. Lack $(2,22)$ or very low $(9)$ activity is found in classical homozygotes and a residual activity of about $3-5 \%$ is usually present in mild permanent hyperphenylalaninemia $(2,17,22)$, whereas the mean $\mathbf{k}$ values obtained, respectively, in classical PKU, atypical PKU, and mild permanent form represent $1.8,3.4$, and $6.2 \%$ of the mean observed in normal subjects (Table 2). If we take into account renal excretion following load of a part of phenylalanine and its main metabolites (oOH-PAA and PPA), results are quite concordant.

Furthermore, like in leucinosis and many other errors of metabolism, our results prove that a $10 \%$ residual activity is enough to carry out the conversion of phenylalanine to tyrosine if there is no exogenous or endogenous load. Finally, in the absence of complementation and on the assumption that $50 \%$ of activity occurs from normal allele and only 0 to $3 \%$ from mutant allele, we can explain that there is no significant difference between phenylalanine plasma level and $\mathrm{P} / \mathrm{T}$ molar ratio in the parents of the three groups of variant. However, it is worth noting that Kaufman et al. (19) have found an hydroxylase activity below $10 \%$ in liver biopsies from parents of three patients with mild permanent hyperphenylalaninemia, suggesting thus that classical heterozygotes could well have, too, less than $50 \%$, because in these subjects and in mild heterozygotes values of $\mathrm{P} / \mathrm{T}$ are similarly elevated. However, there is no direct evidence to think that these findings involve a negative interallelic complementation and we must keep in mind that Woolf et al. (28) and Bremer and Neumann (6) have found a disappearance rate exactly twice as slow in classical heterozygotes than in normal subjects.

Transient PKU has not been precisely described so far. It seems that it was first recognized by Stephenson and McBean (27) and only a few cases have been reported $(5,10)$. This variant is usually mistaken for a classical or atypical PKU, but it differs from them by an increase with time of the phenylalanine tolerance so that, at about 6 months of age, phenylalanine plasma level does not exceed 0.2 or $0.3 \mu \mathrm{mol} \cdot \mathrm{ml}^{-1}$ on a regular diet $(3 \mathrm{~g}$ protein $/ \mathrm{kg}$ body wt). Furthermore, when parents are investigated, neither elevated fasting phenylalanine plasma level nor $\mathrm{P} / \mathrm{T}$ molar ratio increase are found (10), in contrast to common variants, allowing the diagnosis to be suggested immediately. It was just in the family described by Stephenson and McBean (27) and in another "atypical" one published by Hudson et al. (16) that Woolf et al. (29) have found that one of both parents had a typical heterozygotes disappearance phenylalanine curve, in accordance with a first order kinetics, and the other one a rate of disappearance independent of phenylalanine concentration, obeying to zero-order kinetics. These latter two have been considered to possess a "third allele" at the phenylalanine hydroxylase locus, coding for an enzyme more susceptible to substrate inhibition. Unfortunately, in neither case could the children be biochemically examined.

A zero-order kinetics was also demonstrated in our three cases of transient PKU and their phenylalanine plasma level came back to initial values in only $48 \mathrm{hr}$, as in mild permanent hyperphenylalaninemia, which demonstrates thus the persistance of a meta-

Table 2. Comparison between kinetics of disappearance in vivo and hydroxylase activity in vitro

\begin{tabular}{lcccc}
\hline & \multicolumn{3}{c}{ Hydroxylase activity } \\
\cline { 3 - 5 } & $\mathrm{k}$ & $\begin{array}{c}\text { Bartholome } \text { et } \\
\text { al., } 1975\end{array}$ & $\begin{array}{c}\text { Kaufman } \text { et al., } \\
\end{array}$ & $\begin{array}{c}\text { Personal } \\
\text { data }\end{array}$ \\
\hline Typical PKU & 1.8 & $0(13)^{2}$ & $0.27(1)$ & $0(4)$ \\
Atypical PKU & 3.4 & & & $1.8(3)$ \\
& & & & $(0.8-2.5)$ \\
Mild type & 6.2 & $2.9(12)^{3}$ & $5.0(3)$ & $4.7(2)$ \\
& & $(1.5-6.2)$ & $(3.6-6.3)$ & $(4.2-5.2)$ \\
\hline
\end{tabular}

\footnotetext{
${ }^{1}$ Values given in percent of the normal values.

${ }^{2}$ Number of cases in parentheses.

${ }^{3}$ Four other cases have residual activity between 8.7 and $34.5 \%$ of the normal.
} 
bolic disorder after apparent recovery and gives evidence against a transitory phenylalanine hydroxylating system immaturity (5). Actually a zero-order kinetics suggests the existence of a constant factor in the expression of the rate equation and may be observed when one intermediate reactant concentration remains constant. To explain protein concentration dependence at $\mathrm{pH} 8$, Huang and Kaufman (15) invoked the existence of an intermediate released during conversion of phenylalanine to tyrosine, which breaks down nonenzymatically and whose accumulation reduces the specific activity of the enzyme by increasing the apparent $K_{m}$ for the cofactor in the absence of phenylalanine hydroxylase-stimulating protein. A structural alteration of phenylalanine hydroxylase-stimulating protein might be, therefore, the primary defect in this variant form, imposing a limiting step on the reaction. This assumption could also explain that, in the absence of load, parents of these children are undistinguishable from normal subjects.

Hyperphenylalaninemia with normal phenylalanine hydroxylase activity is an heterogenous condition. Two types, at least, are nowadays described: the dihydropteridine reductase deficiency (18) and the defect in biopterin synthesis (20). A variant without alteration of any known component of phenylalanine-hydroxylating system, including phenylalanine hydroxylase, dihydropteridine reductase, and biopterin, has also been reported (3). Among the two cases we have studied, dihydropteridine reductase deficiency has been proved, in one child, by enzymatic assay on needle liver biopsy and fibroblasts, and in the other one, a defect of pterin metabolism was demonstrated by the low serum and urine biopterin derivatives levels (20), phenylalanine hydroxylase and dihydropteridine reductase being normal in the liver (22). In both, the in vivo metabolism of the injected phenylalanine was found, as expected, to follow a first order kinetics. The relative value of the rate constant $(3-4 \%)$ is of the same order of magnitude than in atypical PKU, which agrees well with their daily tolerance. Furthermore, phenylalanine challenge has shown a complete absence of increase of biopterin levels in the case where metabolic defect of biopterin was suspected, whereas in the patient affect by dihydropteridine reductase deficiency it reaches values three to four times higher than those observed in untreated PKU (23). In any case, in the absence of enzymatic assays, an increase of plasma level and urinary excretion of biopterin derivatives in a child suffering from "phenylketonuria resistant to dietary treatment" is very symptomatic of dihydropteridine reductase deficiency.

\section{REFERENCES AND NOTES}

1. Auerbach. V. H., Di George. A. M.. and Carpenter. G. G.: Phenylalaninemia. A study of the diversity of disorders which produce elevation of blood concentration of phenylalanine. In: W. L. Nyhan: Amino Acid Metabolism and Genetic Variation. Ist ed.. p. II (McGraw Hill. Inc.. New York. 1967).

2. Bartholomé. K.. Lutz. P.. and Bickel. H.: Determination of phenylalanine hydroxylase activity in patients with phenylketonuria and hyperphenylalaninemia. Pediatr. Res., 9: 899 (1975).

3. Bartholomé. K.. Byrd. D. J.. Kaufman. S.. and Milstien. S.: Atypical phenylketonuria with normal phenylalanine hydroxylase and dihydropteridine reductase activity in vitro. Pediatrics. 59: 757 (1977).

4. Blaskovics. M. E.. and Shaw. K. N. F.: Hyperphenylalaninemia: methods for differential diagnosis. In: H. Bickel. F. P. Hudson and L. I. Woolf: Phenylketonuria and some other Inborn Errors of Amino Acid Metabolism. p. 98 (Georg Thieme Verlag, Stuttgart, 1971).

5. Bremer, H. J.: Transitory hyperphenylalaninemia. In: H. Bickel, F. P. Hudson and L. 1. Woolf: Phenylketonuria and some other Inborn Errors of Amino Acid Metabolism, p. 93 (Georg Thieme Verlag, Stutgart, 1971).
6. Bremer. H. J.. and Neumann. W.: Tolerance of phenylalanine after intravenous administration in phenylketonuria. heterozygous carriers. and normal adults. Nature (Lond.). 209: 1148 (1966).

7. Cohen. B. E.. Szeinberg. A.. Berman. W.. Aviad. Y.. Crispin. M.. Hirshorn. N.. and Golan. R.: Mental retardation in a family with phenylketonuria and mild hyperphenylalaninemia. Pediatrics, 44: 655 (1969).

8. Efron, M. L.: Classical and mediterranean phenylketonuria. In: W. L. Nyban Amino Acid Metabolism and Genetic Variation, 1st ed., p. 115 (McGraw Hill, Inc., New York, 1967).

9. Friedman. P. A.. Fisher. D. B.. Kang. E. S.. and Kaufman. S.: Detection of hepatic phenylalanine 4-hydroxylase in classical phenylketonuria. Proc. Natl. Acad. Sci. U.S.A.. 70: 552 (1973).

10. Griffin. R. F.. and Elsas, L. J.: Classic phenylketonuria: diagnosis through heterozygote detection. J. Pediatr., 86: 512 (1975).

11. Güttler. F., and Rosleff. F.: Urinary phenylalanine excretion in hyperphenylalaninemic children. Acta Paediatr. Scand., 62: 333 (1973).

12. Güttler, F., and Wamberg. E.: Persistent hyperphenylalaninemia. Acta Paediatr. Scand.. 61: 321 (1972).

13. Hill, J. B.. Summer. G. K.. Pender. M. W.. and Roszel. N. O.: An automated procedure for blood phenylalanine. Clin. Chem.. 11:541 (1965).

14. Hjalmarsson. O.. Jagenburg. R.. and Rödjer. S.: Mild and severe PKU. Comparative studies in two infants. Acta Paediatr. Scand., 60: 11 (1971).

15. Huang. C. Y.. and Kaufman. S.: Studies on the mechanisms of action of phenylalanine hydroxylase and its protein stimulator. I. Enzyme concentration dependence of the specific activity of phenylalanine hydroxylase due to a nonenzymatic step. J. Biol. Chem., 248: 4242 (1973).

16. Hudson. F. P.. Dickinson, R. A.. and Ireland. J. T.: Experiences in the detection and treatment of phenylketonuria. Pediatrics. 31: 47 (1963).

17. Kang. E. S.. Kaufman. S., and Gerald. P. S.: Clinical and biochemical observations of patients with atypical phenylketonuria. Pediatrics, 45: 83 (1970).

18. Kaufman. S.. Holtzman. N. A.. Milstein, S., Butler, I. J.. and Krumholz, A.: Phenylketonuria due to a deficiency of dihydropteridine reductase N. Engl. J. Med.. 293: 785 (1975).

19. Kaufman. S., Max. E. E., and Kang. E. S.: Phenylalanine hydroxylase activity in liver biopsies from hyperphenylalaninemia heterozygotes: deviation from proportionality with gene dosage. Pediatr. Res., 9: 632 (1975).

20. Leeming, R. J., Blair. J. A., and Rey. F.: Biopterin derivatives in atypical phenylketonuria. Lancet, $i: 99$ (1976).

21. Lund, E., and Ovnbøl, A.: Occult phenylketonuria, experience with the Guthrie test. Acta Pathol. Microbiol. Scand., 67: 9 (1966).

22. Rey, F., Blandin-Savoja, F., and Rey, J.: Atypical phenylketonuria with normal dihydropteridine reductase activity. N. Engl. J. Med., 295: 1138 (1976).

23. Rey. F., Harpey. J. P., Leeming. R. J.. Blair. J. A.. Aicardi. J.. and Rey. J.: Les hyperphénylalaninémies avec activité normale de la phénylalanine hydroxylase. Le déficit en tétrahydrobioptérine et le déficit en dihydroptéridine réductase. Arch. Fr. Pediatr., 34: CIX (1977).

24. Rey, F., Pellié, C., Sivy, M., Blandin-Savoja, F., Rey, J., Frézal, J.: Influence of age on ortho-hydroxyphenylacetic acid excretion in phenylketonuria and its genetic variants. Pediatr. Res., 8: 540 (1974).

25. Schneider, A. J.: The kinetics of phenylalanine disposal in PKU and PKU variants. (abstract). Pediatr. Res., 7: 394 (1973).

26. Smith. I.. Clayton. B. E.. and Wolff. O. H.: New variant of phenylketonuria with progressive neurological illness unresponsive to phenylalanine restriction. Lancet. $i$ : 1108 (1975).

27. Stephenson. J. B. P., and McBean. M. S.: Diagnosis of phenylketonuria (phenylalanine hydroxylase deficiency temporary and permanent). Br. Med. J.. 3: 579 (1967).

28. Woolf, L. I.. Cranston, W. I., and Goodwin, B. L.: Genetics of phenylketonuria. Nature (Lond.) 213: 882 (1967).

29. Woolf. L. I.. Goodwin. B. L.. Cranston. W. I.. Wade. D. N.. Woolf. F., Hudson. F. P., and McBean. M. S.: A third allele at the phenylalanine-hydroxylase locus in mild phenylketonuria (hyperphenylalaninaemia). Lancet. $i$ : 114 (1968).

30. Yu. J. S. and O'Halloran. M. T.: Atypical phenylketonuria in a family with a phenylketonuric mother. Pediatrics. 46: 707 (1970).

31. Technicon AutoAnalyzer, Technicon Corporation, Domont, France.

32. This research was supported by a research grant (ATP:37.76.69) from the Institut National de la Santé et de la Recherche Médicale (INSERM).

33. Requests for reprints should be addressed to: F. Rey. M. D.. Unité de Recherches de Génétique Médicale. Hôpital des Enfants-Malades. 149 Rue de Sèvres. 75730 Paris Cedex 15. France.

34. Received for publication October 10, 1977

35. Accepted for publication March 28. 1978. 